\title{
Electrophysiological Assessment of the Deltoid Muscle after Minimally Invasive Treatment of Proximal Humerus Fractures - A Clinical Observation
}

\author{
Götz Röderer ${ }^{*}, 1$, Anne-Dorte Sperfeld ${ }^{2}$, Philipp Hansen ${ }^{1}$, Gert Krischak ${ }^{3}$, Florian Gebhard ${ }^{1}$ and \\ Jan Kassubek ${ }^{4}$ \\ ${ }^{I}$ University of Ulm, Department of Orthopaedic Trauma, Steinhövelstraße 9, D-89075 Ulm, Germany \\ ${ }^{2}$ Department of Neurology, HELIOS Hospital Bad Saarow, Germany \\ ${ }^{3}$ University of Ulm, Institute for Rehabilitation Sciences, Germany \\ ${ }^{4}$ University of Ulm, Department of Neurology, Germany
}

\begin{abstract}
The minimal anterolateral acromial approach offers a less invasive access to the proximal humerus. Functional impairment following this procedure may be caused by paresis of the deltoid muscle as a result of iatrogenic injury to the axillary nerve. It was addressed whether electromyography (EMG) of the deltoid muscle gives evidence for an axillary nerve lesion in association with the minimal anterolateral acromial approach.

Twenty-three patients (14 men, 9 women; average age 58 years) with proximal humerus fractures were included in this clinical observation. Follow-up was performed 6 weeks $(6 \mathrm{w}), 6$ months $(6 \mathrm{~m})$ and 12 months $(12 \mathrm{~m})$ postoperatively. EMG changes indicating either lesion of the axillary nerve or direct muscle trauma were distinguished in "acute", "chronic" and "combined" and semi quantified in "slight", "moderate" and "severe". Patients were examined clinically (standard neurological examination and Constant Score).

Three cases of incomplete axillary nerve lesion with limited functional impairment were detected. Subclinical EMG signs of neural impairment of the deltoid muscle were observed frequently $(6 \mathrm{w}, \mathrm{N}=8 ; 6 \mathrm{~m}, \mathrm{~N}=8 ; 12 \mathrm{~m}, \mathrm{~N}=7)$. Functional outcome did not show an association with EMG.

Most patients presented with subclinical and most likely trauma- related neurogenic lesions of the deltoid muscle following the anterolateral acromial approach. Despite the fact that the axillary nerve does not function normally following this less-invasive approach for fixation of proximal humerus fractures, this does not appear to affect the clinical outcome. Prospective studies with larger sample sizes are required to determine the effect of axillary nerve retraction in the more commonly used deltopectoral approach.
\end{abstract}

Keywords: Proximal humerus, axillary nerve, fracture, minimal invasive, EMG.

\section{INTRODUCTION}

Avascular necrosis (AVN) and non-union are common complications of proximal humerus fractures, caused by vascular insult to the critical blood supply of the humeral head by the trauma itself and/or surgical dissection [1]. Traditional plating techniques using large surgical approaches with extensive soft tissue manipulation promote this problem of vascular insult by causing devascularisation to the fracture fragments with subsequent delayed healing, or AVN of the humeral head [2, 3]. Minimally invasive (MI) techniques have been developed to address this problem by avoiding major disruption of the local blood supply [4-7]. Several studies report up to threefold lower rates of AVN in MI compared to open surgical techniques [8-10]. The earlier use closed or percutaneous fracture reduction which causes less impairment of the periosteal blood supply. However, not every fracture is eligible for percutaneous reduction [11-13].

*Address correspondence to this author at the University of Ulm, Department of Orthopaedic Trauma, Steinhövelstraße 9, D-89075 Ulm, Germany; Tel: +49 731 50054565; Fax: +49 731 50054502;

E-mail: goetz.roederer@uniklinik-ulm.de
The minimal anterolateral acromial approach allows for a less invasive access to the proximal humerus for MI fracture treatment [5]. Due to the close anatomical relationship to the axillary nerve there is the risk of nerve lesion with reports in the literature that the minimal anterolateral acromial approach might cause an affectation of the anterior portion of the deltoid muscle as a result of a lesion to the axillary nerve [14-16]. In this clinical observation, it was investigated whether neurological examination and EMG show symptoms and signs of lesion to the anterior portion of the deltoid muscle following MI fracture treatment using the minimal anterolateral approach and what functional impairment is caused by it.

\section{MATERIAL AND METHODOLGY}

\section{Surgical Technique and Patient Collective}

Patients who had sustained a fracture of the proximal humerus treated by use of the minimal anterolateral acromial approach and who underwent neurological examination including EMG during routine follow-up were evaluated (Fig. 1) [15]. Informed consent to collect the existing data was obtained. 


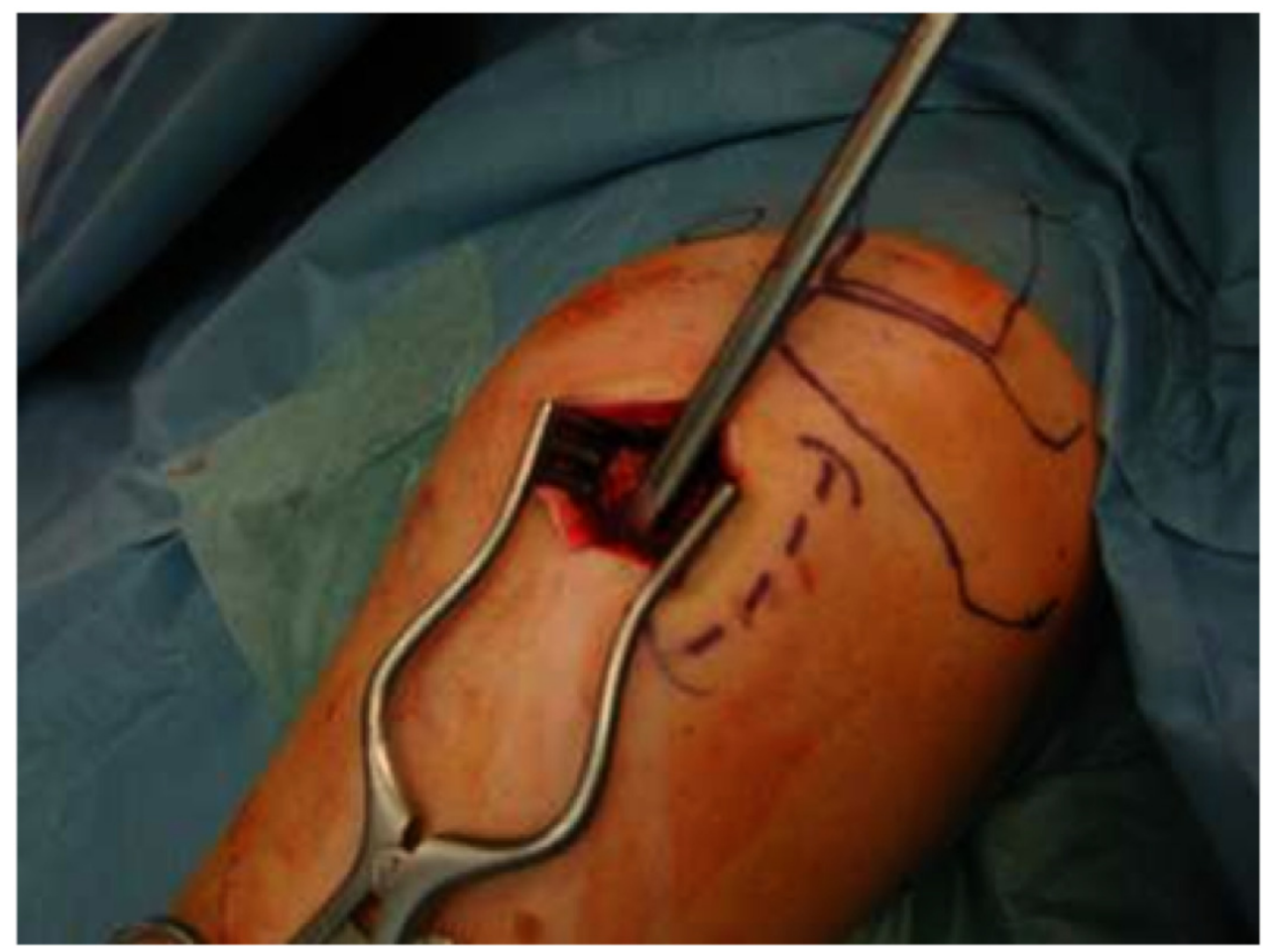

Fig. (1). Minimal anterolateral approach to a left proximal humerus. The anatomical landmarks (clavicle, acromion) have been marked. The dotted line marks the lateral aspect of the deltoid muscle, the incision runs anterior to it.

Along with the individual clinical condition of the patient, the indication for surgery was based on the Neer criteria, i.e. fragment dislocation of $>1 \mathrm{~cm}$ or $>0.5 \mathrm{~cm}$ for the greater tuberosity, shaft displacement of $>10 \mathrm{~mm}$, angular displacement of $>45^{\circ}$, destruction of the medial metaphyseal column, and an intraarticular step off of $>2 \mathrm{~mm}$ $[17,18]$. Patients with pathological fractures, open fractures and neurological or metabolic diseases were not included. Fractures were treated with percutaneous reduction and locked plating (NCB-PH ${ }^{\circledR}$, Zimmer IN-USA) [5]. According to the criteria given above, we were able to analyze the data of 23 patients ( 12 women, 11 men; average 58 years, range 28 - 90) who were operated between October 2005 and July 2007 (22 months). According to the Orthopaedic Trauma Association (OTA) classification system there were $\mathrm{N}=7$ type A, $\mathrm{N}=9$ type $\mathrm{B}$ and $\mathrm{N}=7$ type $\mathrm{C}$ fractures [19]. The data of ten patients ( 7 women, 3 men; average age 67 years, range $42-89$ ) with conservatively treated proximal humerus fractures and who also underwent neurological evaluation including EMG due to supposed axillary nerve lesion during routine follow-up served as a control group $(\mathrm{N}=6$ OTA type $\mathrm{B}, \mathrm{N}=4$ OTA type A).

\section{Follow-Up}

The data of ten postoperative follow-up visits after 6 weeks $(6 \mathrm{w}), 6(6 \mathrm{~m})$ and $12(12 \mathrm{~m})$ months, respectively were evaluated. Data of the control group were drawn from a follow-up visit 6 weeks after the trauma.

At each time point, as part of the routine follow-up, the patients were investigated clinically and by EMG, i.e. standardized neurological examination including motor and sensory function was done (JK, ADS). The Medical Research Council (MRC) Scale was used in order to graduate the muscle strength [20]. EMG of the deltoid muscles following standardized clinical procedures was performed by a board-certified neurologist (Multiliner, Würzburg, Germany) - for the needle localization, please cf. also Fig. (2). With respect to the presentation and the extent of the findings, EMG changes were described as "acute" and "chronic" neurogenic changes and semi-quantified in "slight", "moderate", and "severe" [21, 22]. Global functional assessment of the affected shoulder was performed using the Constant Score [23]. Visual analogue scale (VAS) was used to assess the subjective outcome for pain $(10$ points $=$ maximum $)$ and mobility $(10$ points $=$ optimal function) [5].

\section{RESULTS}

\section{Surgical Group}

At $6 \mathrm{w}$, EMG showed signs of neurogenic impairment of different severity in all patients (slight, $\mathrm{N}=6$; moderate, $\mathrm{N}=$ 4). In two patients with slight impairment, clinical examination demonstrated hypaesthesia in the axillary nerve area, deltoid muscle paresis MRC 4/5 and (in one case) beginning muscle atrophy. These cases were consistent with incomplete axillary nerve lesion. At $6 \mathrm{~m}$, there were nine cases with EMG abnormalities (slight, $\mathrm{N}=6$; moderate, $\mathrm{N}=$ 2 ; severe, $\mathrm{N}=1$ ), among whom one of the patients with incomplete axillary nerve lesion at $6 \mathrm{w}$ now reported improved hypaesthesia (EMG: slight denervation). The patient with severe signs of denervation (acute and chronic) presented clinical findings of an incomplete axillary nerve lesion (hypaesthesia, MRC 1-2/5 and muscle atrophy). At $12 \mathrm{~m}$, there were seven cases with pathological EMG findings (slight, $\mathrm{N}=4$; moderate, $\mathrm{N}=2$; severe, $\mathrm{N}=1$ ). Clinical investigation of the sensomotor function of the 
axillary nerve was uneventful in all of these patients. In summary, there were three cases of incomplete axillary nerve lesion confirmed by clinical investigation and EMG (Table 1).

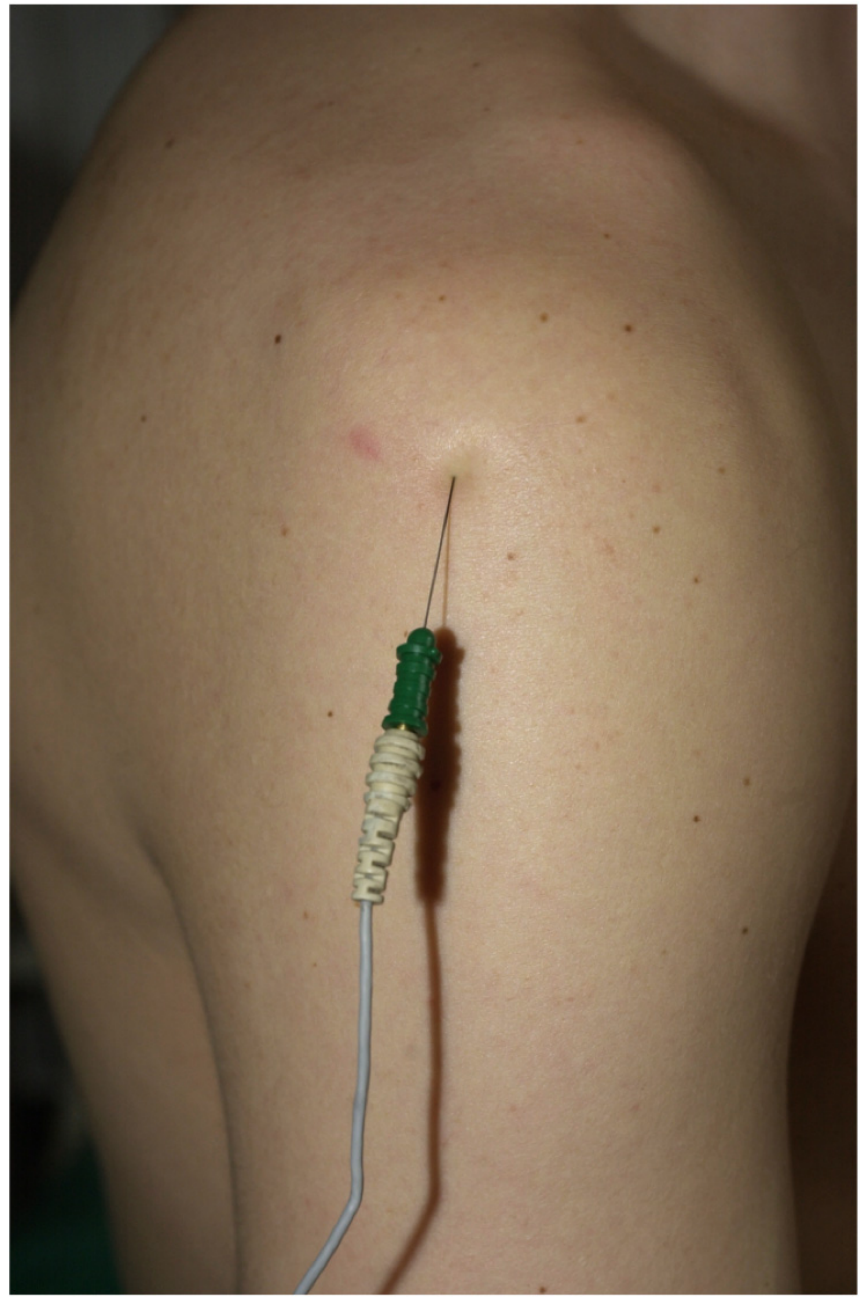

Fig. (2). Fine needle EMG of the deltoid muscle (posterior portion, right arm).
Patients without any EMG signs of neurogenic impairment $(\mathrm{N}=4)$ had no hypaesthesia and a normal muscle function. Subclinical EMG signs were seen in $\mathrm{N}=21$ patients. One patient (age 66, female), who complained about pain (VAS 6 and 5) had MRC of 3-4/5 at both of her follow-up appointments $(6 \mathrm{w}$ and $6 \mathrm{~m})$. Muscle atrophy was found in seven patients within the group of patients with EMG abnormalities. However, this was also noted in three patients without any EMG signs of neurogenic impairment (Table 1). Out of all EMG investigations of the surgically treated patients $(\mathrm{N}=30), 18$ showed impairment of both the anterior and the posterior portion of the deltoid muscle. In seven patients, EMG demonstrated exclusive abnormalities of the anterior portion, while the posterior portion was intact. An isolated impairment of the posterior portion was found in one patient.

Longitudinal assessment was obtained in seven cases with two follow-up appointments. Here, EMG signs of neurogenic impairment improved or even fully resolved $(\mathrm{N}=$ 3 ) or turned from acute into chronic of the same severity ( $\mathrm{N}$ $=3$ ). Among the earlier was one of the patients with incomplete axillary nerve lesion, in whom sensomotor function and EMG signs fully resolved (investigation 25 and 26, Table 1). One patient presented acute neurogenic impairment of the same severity (slight) at both follow-up appointments. This was another patient with incomplete axillary nerve lesion showing hypaesthesia at $6 \mathrm{w}$ investigation, which then had declined at $6 \mathrm{~m}$ investigation (investigation 28 and 29, Table 1).

Constant Score did not show a clear association with EMG findings, but a trend to a lower total score with increasing severity of denervation. Despite persisting pathological EMG signs, there was an improvement in the Constant Score over time (Fig. 3). The subjective outcome by VAS showed a decline of pain and an improvement of mobility over time.

\section{Control Group}

In the conservatively treated group, two patients (age 56 and 89 years, both female) showed slight or moderate

\section{Constant Score}

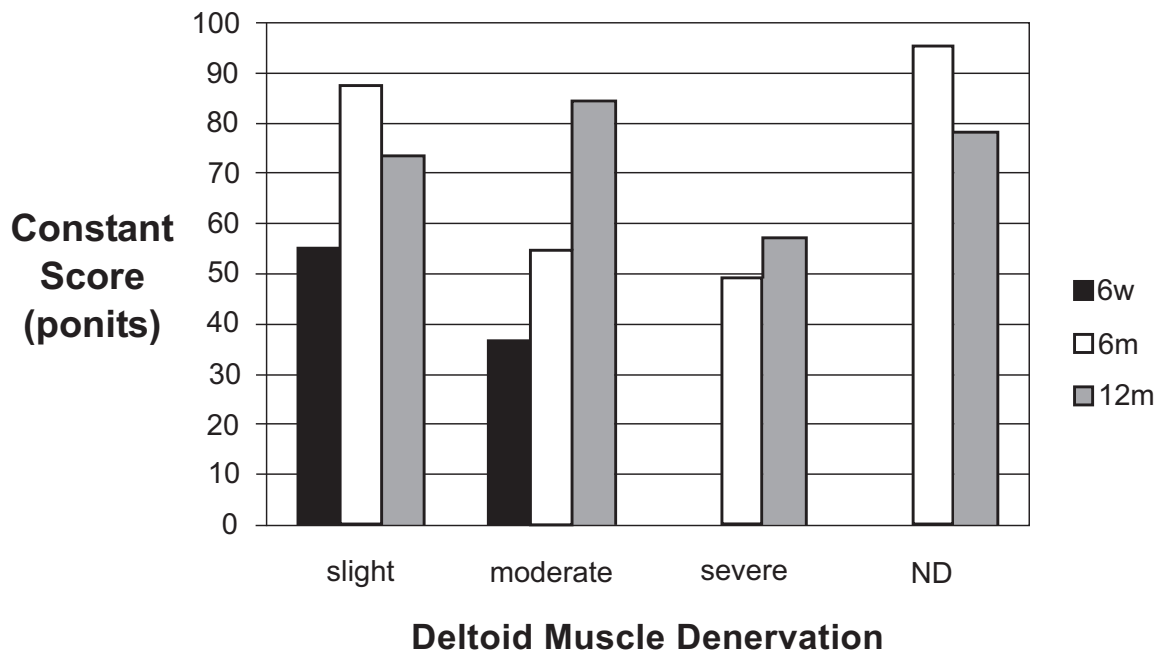

Fig. (3). Constant Score (ND = nothing detected). 
Table 1. EMG (+/- = Signs/No Signs of Deltoid Muscle Denervation), MRC (Medical Research Council) Scale, Sensory Deficit (y/n $=$ Yes/No) and Deltoid Muscle Atrophy $(\mathbf{y} / \mathbf{n}=$ Yes/No). The Highlighted Areas Represent Patients with Axillary Nerve Lesion $(*=$ Incomplete Axillary Nerve Lesion). The Right Column Summarizes the Longitudinal Follow-Up When Available ( $\uparrow=$ Better, $\downarrow=$ Worse, $\leftrightarrow=$ Same; $\mathbf{N}=$ Normal).

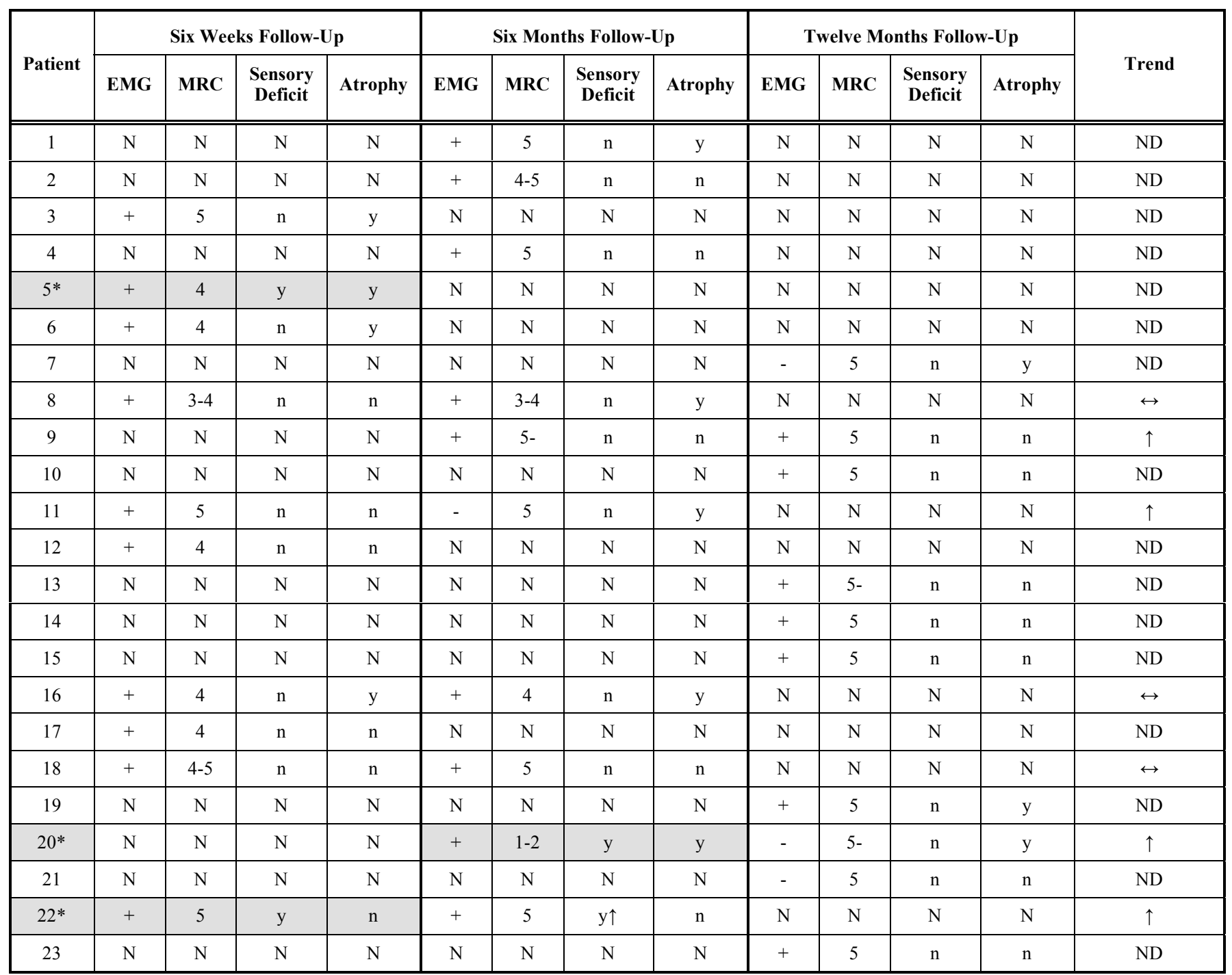

(chronic) EMG signs of neurogenic impairment. Except for muscle atrophy in the older patient, the clinical neurological assessment was uneventful. In the remaining control patients both EMG and clinical investigation revealed normal findings.

\section{DISCUSSION}

The anatomical relationship to the axillary nerve in MI surgical treatment of proximal humerus fractures using the minimal anterolateral acromial approach is close, and there is the risk of nerve lesion [15]. Clinical studies investigating patients being treated with this procedure reported functional shoulder impairment and postulated that this might be the result of iatrogenic injury to the axillary nerve $[5,14,16]$. To our knowledge, we report the first clinical observation with an investigation of the axillary nerve function both cliniconeurologically and electro physiologically after MI fracture treatment of the proximal humerus using a minimal anterolateral acromial approach to verify or falsify this hypothesis.

There were three cases of incomplete axillary nerve lesion in our sample. One of these patients showed improvement, another showed full restitution of the clinical and / or EMG signs during follow up. Our results demonstrate that axillary nerve abnormalities are commonly caused by the minimal anterolateral acromial approach during MI treatment of proximal humerus fractures, but there appears to be little clinical evidence that this affects the long term outcome. Two recent studies report no case of axillary nerve lesion after MI treatment of proximal humerus fractures using clinical assessment only [6, 16]. Two multicenter studies report $2-3 \%$ rates of nerve lesion after both MI and open surgical treatment, again using only clinical investigation $[24,25]$. The low sensitivity of clinical evaluation of axillary nerve function has been described before [26]. Additionally, in patients with proximal humerus fractures the postoperative situation with pain and 
discomfort has to be considered as well as the fact that these patients are usually of old age. As a consequence, it cannot be excluded that clinical evaluation misses cases of axillary nerve lesion, and additional use of EMG as a highly sensitive method therefore reveals higher rates.

Studies using EMG to evaluate the axillary nerve function after proximal humerus fracture or shoulder dislocation are rare and include conservatively treated patients only $[21,22,26]$. The authors report rates of neurogenic impairment of the deltoid muscle between 53 and $58 \%$ as a consequence of the sustained trauma. We also consider most of the observed EMG abnormalities rather as a trauma-related impairment of the muscle than an iatrogenic lesion of the axillary nerve following the surgical procedure. This is supported by both the majority of our patients showing subclinical EMG abnormalities, and the conservatively treated group in which we found two cases (20\%) with EMG signs of neurogenic muscle impairment. Moreover, 19 of the surgically treated patients presented with impairment of the posterior portion of the deltoid muscle, which is not addressed by the surgical approach. The low rate of neurogenic muscle impairment in our control group compared to the literature might be explained in first place by the small sample size and patient selection (i.e. the indirect trauma of the shoulder in all cases due to a fall on the mostly stretched arm). Additionally, the patients were investigated slightly later (six weeks) than in the study referenced (3-4 weeks) [22]. It can't be excluded that a certain degree of recovery takes place during this period of time.

Regarding the functional outcome of the affected shoulder (Constant-Score), no association with EMG could be observed, i.e. patients with normal EMG did not necessarily have the best functional results. Factors like fracture type, quality of fracture reduction or positioning of the implant which may influence the function of the shoulder have to be considered. It was also found that, despite persisting EMG signs of neurogenic impairment of the deltoid muscle of the same severity the results of the Constant Score improved during follow-up. This emphasizes the subclinical character of most EMG abnormalities in our sample. Functional regain rather depended on the time course, i.e. the period of time between surgery and followup, the better the functional results will be, as a well-known and frequently described fact in the orthopedic literature [5, $7,24,25]$. The time course has also an impact on EMG due to reinnervation as seen in most patients in our study with longitudinal follow-up.

With respect to the missing association between the Constant Score and the EMG results, it has to be considered that this score evaluates the global function of the shoulder which obviously can be affected by a number of factors as detailed above. Therefore, additional scores, i.e. DASH Score, should be performed in future studies. In the present preliminary observation, however, the authors used the Constant Score since it is commonly-used in studies on proximal humerus fractures which therefore was considered to alleviate a comparison with the literature.

Further limitations include the low number of patients in both groups. Sample size has to be extended to draw more definitive conclusions from the results and to clarify if there is a correlation between the surgical procedure, EMG findings, and functional results. Also, the influence of the fracture type on the outcome measurements used in the present study has to be addressed in a larger sample size since it is well-known that more complex fracture patterns frequently result in a poor functional outcome without any concomitant injuries, e.g. nerve lesions, due to the severity of the bony lesion. Longitudinal assessment could be obtained only in a very limited number of patients and the follow-up period was quite short which is why the exact impact of time course, i.e. rehabilitation, on the axillary nerve function could not be fully clarified in the present study.

\section{CONCLUSION}

Most patients present subclinical and most likely traumarelated neurogenic impairment of the deltoid muscle in EMG following the anterolateral acromial approach for minimally invasive fracture treatment of the proximal humerus that was not routinely seen in a conservatively treated control group. Only cases of incomplete axillary nerve lesion with limited functional impairment and the potential of full restitution both clinically and electro physiologically were observed. Despite the fact that the axillary nerve does not function normally following a less-invasive approach for fixation of proximal humerus fractures, this does not appear to affect the clinical outcome. Further studies will be necessary to determine the effect of retraction on the axillary nerve in the more commonly used deltopectoral approach.

\section{REFERENCES}

[1] Szyszkowitz R, Seggl W, Schleifer P, Cundy PJ. Proximal humeral fractures: management techniques and expected results. Clin Orthop Relat Res 1993; 292: 13-25.

[2] Wijgman AJ, Roolker W, Patt TW, Raaymakers EL, Marti RK. Open reduction and internal fixation of three and four-part fractures of the proximal part of the humerus. J Bone Joint Surg Am 2002; 84-A: 1919-25.

[3] Gardner MJ, Voos JE, Wanich T, Helfet DL, Lorich DG. Vascular implications of minimally invasive plating of proximal humerus fractures. J Orthop Trauma 2006; 20: 602-7.

[4] Resch H, Hubner C, Schwaiger R. Minimally invasive reduction and osteosynthesis of articular fractures of the humeral head. Injury 2001; 32(Suppl 1): SA25-32.

[5] Röderer G, Gebhard F, Erhardt J, et al. The Non-Contact Bridging Plate. A new fixed-angle device for the minimally-invasive treatment of fractures of the proximal humerus--technique and preliminary results. Unfallchirurg 2007; 110: 505-12.

[6] Laflamme GY, Rouleau DM, Berry GK, et al. Percutaneous humeral plating of fractures of the proximal humerus: results of a prospective multicenter clinical trial. J Orthop Trauma 2008; 22: 153-8.

[7] Röderer G, Erhardt J, Graf M, Kinzl L, Gebhard F. Clinical results for minimally invasive locked plating of proximal humerus fractures. J Orthop Trauma 2010; 24: 400-6.

[8] Kristiansen B, Christensen SW. Plate fixation of proximal humeral fractures. Acta Orthop Scand 1986; 57: 320-3.

[9] Sturzenegger M, Fornaro E, Jakob RP. Results of surgical treatment of multifragmented fractures of the humeral head. Arch Orthop Trauma Surg 1982; 100: 249-59.

[10] Bäthis H, Tingart M, Bouillon B, Tiling T. Surgical treatment of proximal humeral fractures. Is the T-plate still adequate osteosynthesis procedure? Zentralbl Chir 2001; 126: 211-6.

[11] Helmy N, Hintermann B. New trends in the treatment of proximal humerus fractures. Clin Orthop Relat Res 2006; 442: 100-8.

[12] Vallier HA. Treatment of proximal humerus fractures. J Orthop Trauma 2007; 21: 469-76. 
[13] Solberg BD, Moon CN, Franco DP, Paiement GD. Surgical treatment of three and four-part proximal humeral fractures. J Bone Joint Surg Am 2009; 91: 1689-97.

[14] Hata Y, Saitoh S, Murakami N, Kobayashi H, Takaoka K. Atrophy of the deltoid muscle following rotator cuff surgery. J Bone Joint Surg Am 2004; 86-A: 1414-9.

[15] Röderer G, Abouelsoud M, Gebhard F, Bockers TM, Kinzl L. Minimally invasive application of the non-contact-bridging (NCB) plate to the proximal humerus: an anatomical study. J Orthop Trauma 2007; 21: 621-7.

[16] Hepp P, Theopold J, Voigt C, et al. The surgical approach for locking plate osteosynthesis of displaced proximal humeral fractures influences the functional outcome. J Shoulder Elbow Surg 2008; 17: 21-8.

[17] Neer CS, 2nd. Four-segment classification of proximal humeral fractures: purpose and reliable use. J Shoulder Elbow Surg 2002; 11: $389-400$.

[18] Resch H. Fractures of the humeral head. Unfallchirurg 2003; 106: 602-17.

[19] Marsh JL, Slongo TF, Agel J, et al. Fracture and dislocation classification compendium - 2007: Orthopaedic Trauma Association Classification, Database and Outcomes Committee. J Orthop Trauma 2007; 21: S1-133.
[20] Medical Research Council. Aids to the examination of the peripheral nervous system. Memorandum No 45 London: Her Majestiy's Stationary Office 1976.

[21] de Laat EA, Visser CP, Coene LN, Pahlplatz PV, Tavy DL. Nerve lesions in primary shoulder dislocations and humeral neck fractures. A prospective clinical and EMG study. J Bone Joint Surg Br 1994; 76: 381-3.

[22] Visser CP, Tavy DL, Coene LN, Brand R. Electromyographic findings in shoulder dislocations and fractures of the proximal humerus: comparison with clinical neurological examination. Clin Neurol Neurosurg 1999; 101: 86-91.

[23] Constant CR, Murley AH. A clinical method of functional assessment of the shoulder. Clin Orthop Relat Res 1987; 214: 1604.

[24] Sudkamp N, Bayer J, Hepp P, et al. Open reduction and internal fixation of proximal humeral fractures with use of the locking proximal humerus plate. Results of a prospective, multicenter, observational study. J Bone Joint Surg Am 2009; 91: 1320-8.

[25] Brunner F, Sommer C, Bahrs C, et al. Open reduction and internal fixation of proximal humerus fractures using a proximal humeral locked plate: a prospective multicenter analysis. J Orthop Trauma 2009; 23: 163-72.

[26] Visser CP, Coene LN, Brand R, Tavy DL. Nerve lesions in proximal humeral fractures. J Shoulder Elbow Surg 2001; 10: 4217.

(C) Röderer et al.; Licensee Bentham Open.

This is an open access article licensed under the terms of the Creative Commons Attribution Non-Commercial License (http://creativecommons.org/licenses/by-nc/3.0/) which permits unrestricted, non-commercial use, distribution and reproduction in any medium, provided the work is properly cited. 